\section{An Analytical Model for Boundary Layer Attenuation of Acoustic Modes in Rigid Circular Ducts With Uniform Flow}

\section{Chenyang Weng, Friedrich Bake}

German Aerospace Center (DLR), Institute of Propulsion Technology, Engine Acoustics, 10623 Berlin, Germany. chenyang.weng@dlr.de

PACS no. 43.20.Bi, 43.20.Hq, 43.20.Mv, 43.28.Py

\begin{abstract}
Summary
An analytical model is derived for the attenuation of fundamental and higher-order (both axisymmetric and non-axisymmetric) acoustic modes caused by viscothermal losses in the acoustic boundary layer. Flow convection effects are included in the modeling by assuming a uniform mean axial velocity profile. The proposed model can be applied to calculate the damping of the acoustic waves propagating in wide circular flow ducts with rigid walls. A simple and effective way of including the mean flow convection in the modeling for the attenuation of the fundamental mode is also suggested.
\end{abstract}

\section{Introduction}

Accurate models for sound attenuation due to viscothermal losses in the acoustic boundary layer are important in many applications. In one- or multi-port measurements, for example, attenuation models for rigid-walled ducts are necessary for determining the sound scattering characteristics over test samples $[1,2,3]$.

Classical attenuation models include the wide-duct models proposed by Kirchhoff [4] for fundamental modes, and by Beatty [5] for all higher-order modes, and the model proposed by Bruneau et al. [6] for higher-order modes in circular ducts with arbitrary size. In these models, flow effects such as mean flow convection are not included. Recent theoretical investigations into the effect of viscosity and mean flow on duct modes include $[7,8,9,10,11]$. For rigid-walled ducts, Dokumaci [10] extended Bruneau et al.'s model for the effect of uniform mean flow. Like Bruneau et al.'s model, Dokumaci's model is in the form of a non-linear dispersion equation, so the attenuation needs to be computed via a root-finding algorithm. In this paper, we will show that in the case of wide duct with a uniform mean flow an analytical attenuation model can be derived.

The proposed model is based on an asymptotic analysis [12] in the limit of a vanishingly thin acoustic boundary layer. In Section 2, an admittance-like acoustic boundary condition is derived based on the asymptotic analysis and the Myers condition [13]. In Section 3, the admittance is applied to derive the analytical attenuation model. In Section 4, the convection effect on the fundamental mode is discussed based on the proposed model; validity of the model in general is also given. The paper is concluded in Section 5 .

Received 17 May 2016,

accepted 15 September 2016.

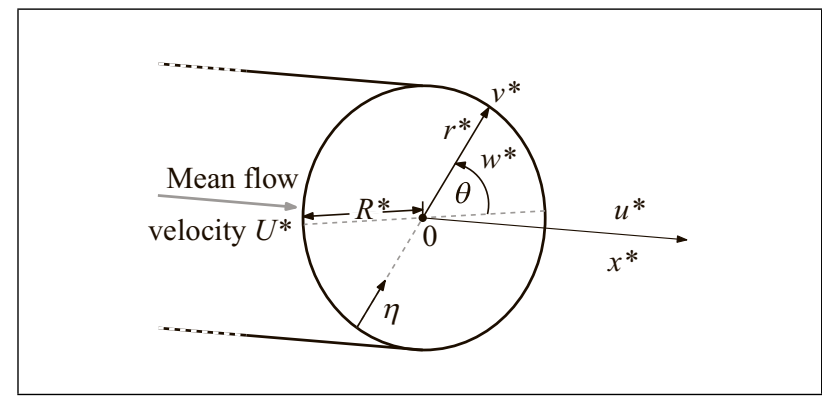

Figure 1. Circular duct geometry with flow.

\section{Asymptotic analyses}

For the convenience of the analysis, the cylindrical coordinate $\mathbf{x}^{*}=\left[x^{*}, r^{*}, \theta\right]$ is used; see Figure 1. We assume time-harmonic waves of the form

$$
\tilde{q}^{*}\left(\mathbf{x}^{*}, t^{*}\right)=\mathbb{R}\left[q^{*}\left(r^{*}, \theta\right) \exp \left(\mathrm{i} \omega^{*} t^{*}-\mathrm{i} k^{*} x^{*}\right)\right],
$$

and make dimensionless as

$$
\begin{aligned}
{[x, r] } & =\left[x^{*}, r^{*}\right] \omega^{*} / c_{0}^{*}, \\
{[p, \mathbf{u}, T] } & =\left[p^{*} / \rho_{0}^{*} c_{0}^{* 2}, \mathbf{u}^{*} / c_{0}^{*}, T^{*} / T_{0}^{*}\right], \\
\Gamma & =k^{*} c_{0}^{*} / \omega^{*}, \\
M & =U^{*} / c_{0}^{*},[v, \chi]=\left[v^{*}, \chi^{*}\right] / v^{*},
\end{aligned}
$$

where $T_{0}^{*}, \rho_{0}^{*}$, and $c_{0}^{*}$ are the reference mean temperature, density, and sound speed, respectively, $\mathbf{u}^{*}=\left[u^{*}, v^{*}, w^{*}\right]$ is the velocity vector defined in Figure $1, \Gamma$ and $M$ denote the dimensionless axial wavenumber and uniform mean axial velocity, respectively, and $\chi^{*}$ is the thermal diffusivity and is herein assumed to be related to the kinematic viscosity via the Prandtl number Pr as $\chi^{*}=v^{*} /$ Pr.

Applying the wave form in the linearized Navier-Stokes equations, one arrives at an equation matrix of an eigenvalue-problem form (cf. [11, equation (2.5)]): $(\mathbb{A}+\Gamma \mathbb{B}) \mathbf{q}=0$, where $\mathbf{q}=$ $[p, u, v, w, T]$, and $\mathbb{A}, \mathbb{B}$ are two-dimensional operators acting on the duct cross-section. At the duct wall, the following impermeable and no-slip conditions are applied

$$
u=v=w=0, \quad \text { for } r=R,
$$

where $R=R^{*} \omega^{*} / c_{0}^{*}$ is the dimensionless duct radius. As for the boundary condition for $T$, two ideal cases are considered in this paper

$$
\left\{\begin{array}{ll}
\text { Conducting wall: } & T=0, \\
\text { Isolating wall: } & \partial T / \partial r=0,
\end{array} \text { for } r=R .\right.
$$

Following Nayfeh [12], we apply the composite expansion to the wave variables as $\mathbf{q}(r, \theta)=\mathbf{q}_{a}(r, \theta)+\mathbf{q}_{b}(\eta, \theta)$, where $\eta=$ $(R-r) / \delta$ is the normal distance from the wall (see Figure 1) scaled by the acoustic boundary layer thickness $\delta=\delta^{*} \omega^{*} / c_{0}^{*}$. Herein $\delta^{*}=\sqrt{\nu^{*} / \omega^{*}}$ is defined as the dimensional boundary layer length scale. The terms with the subscript " $a$ " and " $b$ " represent the acoustic field and the boundary layer field, respectively.

The acoustic field $\mathbf{q}_{a}$ represents waves that propagate isentropically in the limit of vanishing acoustic boundary layer thickness. Inserting the composite expansion into the equation matrix of $\mathbf{q}$, taking the limit of $\delta \rightarrow 0$, and collecting terms related to $\mathbf{q}_{a}$, one 
can show that the acoustic field is governed by the convective wave equation

$$
\frac{\partial^{2} p_{a}}{\partial r^{2}}+\frac{1}{r} \frac{\partial p_{a}}{\partial r}+\frac{1}{r^{2}} \frac{\partial^{2} p_{a}}{\partial \theta^{2}}+\Gamma_{\theta r}^{2} p_{a}=0,
$$

where $\Gamma_{\theta r}$ is the cross-section wavenumber and is given by $\Gamma_{\theta r}^{2}=$ $\Omega^{2}-\Gamma^{2}$, and $\Omega=1-M \Gamma$. The normalized acoustic pressure is given by the general solution to Equation (3) as

$$
p_{a}=\exp (\mathrm{i} m \theta) J_{m}\left(\Gamma_{\theta r} r\right),
$$

where $m$ is the azimuthal mode order and is an integer number, and $J_{m}$ is the $m$ th order Bessel function of the first kind. The other acoustic variables are related to the pressure as

$$
\text { and } \begin{aligned}
{\left[u_{a}, v_{a}, w_{a}\right] } & =\left[\Gamma, \mathrm{i} \frac{\partial}{\partial r}, \frac{\mathrm{i}}{r} \frac{\partial}{\partial \theta}\right] \frac{p_{a}}{\Omega} \\
T_{a} & =(\gamma-1) p_{a},
\end{aligned}
$$

where $\gamma$ is the heat capacity ratio.

The boundary layer field $\mathbf{q}_{b}$ is generated near the wall by diffusions of momentum and heat, and the diffusion distance is characterized by $\delta$. In the limit of $\delta \rightarrow 0$, the boundary layer field vanishes as $\eta \rightarrow \infty$. Under such a limit, the radial velocity $v_{b}=\mathcal{O}(\delta)$ assumes very small values, therefore a boundary layer transformation $\hat{v}_{b}=v_{b} / \delta$ is applied to balance the mass flux [14, ch. 6]. Following a procedure similar to deriving the acoustic field equation, one can obtain the boundary layer equations in the vicinity of $r=R$ (cf. [9, equations (2.4a-e)])

$$
\begin{aligned}
& \mathrm{i} \gamma \Omega p_{b}-\mathrm{i} \Omega T_{b}-\mathrm{i} \Gamma u_{b}-\frac{\partial \hat{v}_{b}}{\partial \eta}+\frac{1}{R} \frac{\partial w_{b}}{\partial \theta}=0, \\
& \mathrm{i} \Omega u_{b}=\mathrm{i} \Gamma p_{b}+\frac{\partial^{2} u_{b}}{\partial \eta^{2}}, \\
& \frac{\partial p_{b}}{\partial \eta}=0, \\
& \mathrm{i} \Omega w_{b}=-\frac{1}{R} \frac{\partial p_{b}}{\partial \theta}+\frac{\partial^{2} w_{b}}{\partial \eta^{2}}, \\
& \mathrm{i} \Omega T_{b}=\mathrm{i} \Omega(\gamma-1) p_{b}+\frac{1}{\operatorname{Pr}} \frac{\partial^{2} T_{b}}{\partial \eta^{2}} .
\end{aligned}
$$

Equation (8) gives $p_{b}=0$ due to the boundary condition at $\eta=$ $\infty$, then equations (7), (9) and (10) give

$$
\left(\frac{\partial^{2}}{\partial \eta^{2}}-\mathrm{i} \Omega\right)\left[u_{b}, w_{b}\right]=0
$$

and $\quad\left(\frac{\partial^{2}}{\partial \eta^{2}}-\mathrm{i} \Omega \operatorname{Pr}\right) T_{b}=0$

The solutions to the above equations that satisfy the boundary conditions at $\eta=\infty$ are

$$
\left[\begin{array}{l}
u_{b} \\
w_{b} \\
T_{b}
\end{array}\right]=\left[\begin{array}{c}
A(\theta) \\
B(\theta) \\
C(\theta)
\end{array}\right] \exp \left\{-\sqrt{\mathrm{i} \Omega}\left[\begin{array}{c}
1 \\
1 \\
\sqrt{\mathrm{Pr}}
\end{array}\right] \eta\right\},
$$

where $A, B$, and $C$ are the wall values, and they are coupled to the acoustic terms via the boundary conditions equations (1) and (2),

$$
\left\{\begin{array}{l}
A=-u_{a}=-\frac{\Gamma}{\Omega} p_{a}, \\
B=-w_{a}=\frac{1}{\mathrm{i} \Omega R} \frac{\partial p_{a}}{\partial \theta}, \\
\text { Conducting wall: } C=-(\gamma-1) p_{a}, \quad \text { for } r=R . \\
\text { Isolating wall: } C=(\gamma-1) \delta \sqrt{\frac{\mathrm{i} \Omega}{\operatorname{Pr}}} v_{a} .
\end{array}\right.
$$

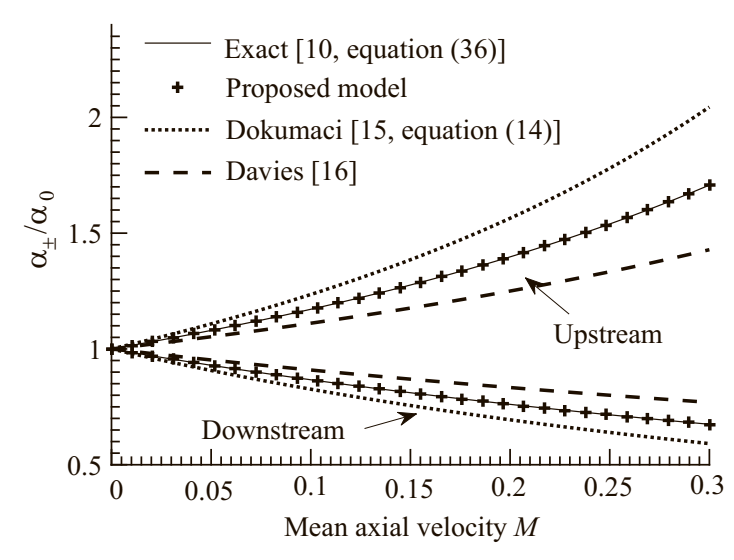

Figure 2. Sound attenuation coefficients $\alpha_{ \pm}$normalized by quiescent values $\alpha_{0}$ for the $(0,0)$ mode at different Mean axial velocities, with shear wavenumber $\mathrm{sh}=500$.

Inserting equations (12) and (13) into Equation (6), integrating from $\eta=0$ to $\infty$, and using $v_{a}(R)=-\delta \hat{v}_{b}(0)$, one gets the following relation for $r=R$,

$$
\begin{aligned}
v_{a}= & \frac{\delta \sqrt{\mathrm{i} \Omega}}{1+\mathrm{i} E \Omega \delta^{2}(\gamma-1) / \operatorname{Pr}} \\
& \cdot\left[\frac{\Gamma^{2}}{\Omega^{2}}+\frac{m^{2}}{(\Omega R)^{2}}+\frac{F(\gamma-1)}{\sqrt{\operatorname{Pr}}}\right] p_{a},
\end{aligned}
$$

where $E=0, F=1$ for conducting walls, and $E=1, F=0$ for isolating walls. In the latter case, the contribution of the thermal boundary layer is $\mathcal{O}\left(\delta^{2}\right)$ and hence negligible compared to unity, then the only difference between these two cases is the presence of the last term $(\gamma-1) / \sqrt{\operatorname{Pr}}$.

Based on the Myers condition [13], a dimensionless acoustic admittance can be derived from Equation (14) as

$Y=\frac{v_{a}}{\Omega p_{a}}=\frac{(1+\mathrm{i}) \delta}{\sqrt{2 \Omega}}\left[1-\frac{\left(\Gamma_{\theta r} R\right)^{2}-m^{2}}{(\Omega R)^{2}}+\frac{\gamma-1}{\sqrt{\mathrm{Pr}}}\right]$

for conducting walls; one can simply drop the last term in Equation (15) to get the admittance for isolating walls.

Equation (15) can also be derived by adapting [7, equation (23)] to rigid-wall circular ducts with uniform flow.

\section{Attenuation model}

The admittance given by Equation (15) allows one to derive an analytical attenuation model for the acoustic field. Let us expand asymptotically about an inviscid duct mode with axial wavenumber $\Gamma^{(0)}$, so that

$$
\Gamma=\Gamma^{(0)}+\delta \Gamma^{(1)}+\mathcal{O}\left(\delta^{2}\right) .
$$

Hence,

$$
\Omega=\Omega^{(0)}-\delta M \Gamma^{(1)}+\mathcal{O}\left(\delta^{2}\right)
$$

and

$$
\Gamma_{\theta r}=\Gamma_{\theta r}^{(0)}+\delta \Gamma_{\theta r}^{(1)}+\mathcal{O}\left(\delta^{2}\right),
$$

with

$$
\begin{aligned}
& \Omega^{(0)}=1-M \Gamma^{(0)}, \quad \Gamma_{\theta r}^{(0) 2}=\Omega^{(0) 2}-\Gamma^{(0) 2}, \\
& \Gamma_{\theta r}^{(1)}=-\Gamma^{(1)}\left(\Gamma^{(0)}+M \Omega^{(0)}\right) / \Gamma_{\theta r}^{(0)} .
\end{aligned}
$$


Since $\Gamma^{(0)}$ is an inviscid duct mode, it satisfies

$$
J_{m}^{\prime}\left(\Gamma_{\theta r}^{(0)} R\right)=0 .
$$

Then the Taylor expansion of $J_{m}^{\prime}$ gives

$$
J_{m}^{\prime}\left(\Gamma_{\theta r} R\right)=\delta \Gamma_{\theta r}^{(1)} R J_{m}^{\prime \prime}\left(\Gamma_{\theta r}^{(0)} R\right)+\mathcal{O}\left(\delta^{2}\right) .
$$

Substituting Equation (4) into Equation (15) leads to

$$
\begin{aligned}
\frac{v_{a}}{\Omega p_{a}} & =\frac{\mathrm{i} \Gamma_{\theta r} J_{m}^{\prime}\left(\Gamma_{\theta r} R\right)}{\Omega^{2} J_{m}\left(\Gamma_{\theta r} R\right)} \\
& =\frac{-\mathrm{i} \delta R \Gamma^{(1)}\left(\Gamma^{(0)}+M \Omega^{(0)}\right) J_{m}^{\prime \prime}\left(\Gamma_{\theta r}^{(0)} R\right)}{\Omega^{(0) 2} J_{m}\left(\Gamma_{\theta r}^{(0)} R\right)}+\mathcal{O}\left(\delta^{2}\right) \\
& =\frac{(1+\mathrm{i}) \delta}{\sqrt{2 \Omega^{(0)}}}\left[1-\frac{\left(\Gamma_{\theta r}^{(0)} R\right)^{2}-m^{2}}{\left(\Omega^{(0)} R\right)^{2}}+\frac{\gamma-1}{\sqrt{\operatorname{Pr}}}\right]+\mathcal{O}\left(\delta^{2}\right) .
\end{aligned}
$$

The above equation can be simplified using Bessel's equation for $J_{m}(z)$, i.e. $z^{2} J_{m}^{\prime \prime}+z J_{m}^{\prime}+\left(z^{2}-m^{2}\right) J_{m}=0$, which gives $J_{m}^{\prime \prime}\left(\Gamma_{\theta r}^{(0)} R\right) / J_{m}\left(\Gamma_{\theta r}^{(0)} R\right)=m^{2} /\left(\Gamma_{\theta r}^{(0)} R\right)^{2}-1$; then, the axial wavenumber perturbation is given by $\Gamma_{ \pm}^{(1)}=(1-\mathrm{i}) \beta_{ \pm}$, where

$$
\beta_{ \pm}=\frac{\Omega^{(0)} \sqrt{\Omega^{(0)}}\left[1-\frac{\left(\Gamma_{\theta r}^{(0)} R\right)^{2}-m^{2}}{\left(\Omega^{(0)} R\right)^{2}}+\frac{\gamma-1}{\sqrt{\mathrm{Pr}}}\right]}{R \sqrt{2}\left(\Gamma_{ \pm}^{(0)}+M \Omega^{(0)}\right)\left[1-\left(\frac{m}{\Gamma_{\theta r}^{(0)}}\right)^{2}\right]}+\mathcal{O}(\delta) .
$$

Here " \pm " represent downstream/upstream propagations relative to the mean flow, and $\Gamma_{ \pm}^{(0)}$ is given by

$$
\Gamma_{ \pm}^{(0)}=\frac{-M \pm \sqrt{1-\left(1-M^{2}\right) \Gamma_{\theta r}^{(0) 2}}}{1-M^{2}} .
$$

All perturbations have $x$ dependence as $\exp \left(-\mathrm{i} \Gamma_{ \pm} x\right)$, and therefore decay as $\exp \left(\mp \mathrm{i} \alpha_{ \pm} x\right)$, where $\alpha_{ \pm}=\mp \Im\left(\Gamma_{ \pm}\right)$is the dimensionless attenuation coefficient. For a cut-on acoustic mode, i.e. when $\Im\left(\Gamma_{ \pm}^{(0)}\right)=0$, the attenuation is given by

$$
\alpha_{ \pm}=\mp \Im\left(\delta \Gamma_{ \pm}^{(1)}\right)= \pm \delta \beta_{ \pm} .
$$

Equation (20) is the primary result of this paper. It reduces to the quiescent-fluid model proposed by Beatty [5] when $M=0$.

\section{Discussion}

\subsection{Convection effects on the $(0,0)$ mode}

For the attenuation of the fundamental $(0,0)$ mode, Equation (20) gives

$$
\begin{aligned}
\alpha_{ \pm} & = \pm \frac{\Omega^{(0)} \sqrt{\Omega^{(0)}}\left(1+\frac{\gamma-1}{\sqrt{\mathrm{Pr}}}\right)}{\operatorname{sh} \sqrt{2}\left(\Gamma_{ \pm}^{(0)}+M \Omega^{(0)}\right)} \\
& =\frac{\alpha_{0}}{(1 \pm M)^{3 / 2}},
\end{aligned}
$$

where sh $=R / \delta$ is the shear wavenumber, and $\alpha_{0}=$ $2^{-1 / 2} \operatorname{sh}^{-1}\left[1+(\gamma-1) \operatorname{Pr}^{-1 / 2}\right]$ is the attenuation coefficient in quiescent fluid which is the same as Kirchhoff's wide-duct model [4]. Equation (21) illustrates an effective way to include uniformflow convection effects in the modeling of the attenuation for the $(0,0)$ mode. Compared with other analytical convection models, such as Dokumaci's simplified model [15, equation (14)], and Davies' model [16] $\alpha_{ \pm}=\alpha_{0} /(1 \pm M)$, the proposed model is more accurate; this is shown in Figure 2 by validating these models by the iteration solutions to the exact dispersion equation for uniform mean flow [10, equation (36)].

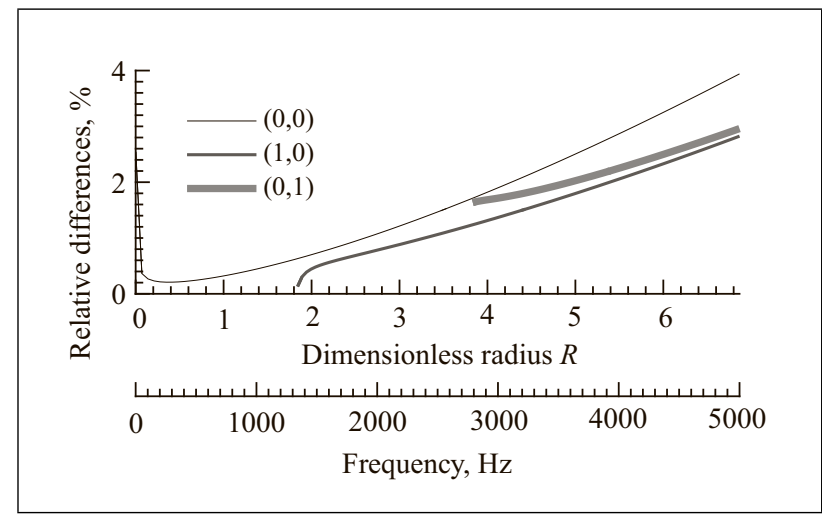

Figure 3. Relative differences between the attenuation model and the exact dispersion equation [10] for the upstream $(0,0),(1,0)$ and $(0,1)$ modes, with $M=0.3$. Duct geometry is taken from a realistic measurement setup [17] with $R^{*}=75 \mathrm{~mm}$. At the highest frequency $\delta \approx 2 \times 10^{-3}$.

\subsection{Validity of the proposed model}

The following three issues need to be considered while using the proposed model.

First, the validity of the proposed model requires $\delta=$ $\sqrt{\nu^{*} \omega^{*}} / c_{0}^{*} \ll 1$ because the asymptotic solution (14) is derived by assuming the $\mathcal{O}(\delta)$ terms to be negligible. For typical multiport measurements with air flows, the maximum dimensional frequency of interest is less than $10 \mathrm{kHz}$, so the maximum of $\delta$ is less than $\approx 3 \times 10^{-3}$ and the underlying assumption is generally valid; therefore, use of the simplified model may only result in small differences from the exact dispersion equation [10] (see Figure 3).

Second, the proposed model is valid for wide ducts (large sh limit) like Kirchhoff's model, because in deriving the boundary layer equations (6)-(10) the coefficient $1 / r$ is approximated by its wall value $1 / R$. Such wide-duct approximation is shown [18] to be valid when sh $\gtrsim 10$. In most multi-port measurements with air flow, the duct radius is larger than $20 \mathrm{~mm}$ and the shear wavenumber at $50 \mathrm{~Hz}$ is then higher than 90 , so the wide-duct condition is well satisfied.

Third, the only flow effect that is included in the proposed model is the mean flow convection. In practice, other flow effects, such as absorption by turbulence, and refraction by mean flow shear, may be significant for the sound propagation under certain conditions and need to be considered (cf. $[7,19,20]$ ).

\section{Conclusions}

The primary contribution of this paper is the proposed attenuation model given by Equation (20). This model incorporates the flow convection effect in Kirchhoff's and Beatty's models, and can be applied to compute the damping of the fundamental and higher-order modes in wide circular flow ducts caused by viscothermal losses in the acoustic boundary layer.

Another contribution is that, an effective way of considering the convection in the modeling of the attenuation for the fundamental $(0,0)$ mode is suggested. As the proposed model shows, the factor $1 /(1 \pm M)^{3 / 2}$, instead of the commonly used factor $1 /(1 \pm M)$, should be applied to quiescent-fluid attenuation coefficients, such as those computed from Kirchhoff's model, to count the convection by a uniform mean axial flow. 


\section{Acknowledgement}

We are grateful to a reviewer (Dr E.J. Brambley) for the direct derivation of the attenuation coefficient in Section 3, which improves upon the energy-based derivation present in a previous draft of this paper.

C. Weng gratefully acknowledges support from the DLRDAAD Research Fellowship.

\section{References}

[1] P. Durrieu, G. Hofmans, G. Ajello, R. Boot, Y. Auregan, A. Hirschberg, M. C. A. M. Peters: Quasisteady aeroacoustic response of orifices. The Journal of the Acoustical Society of America 110 (2001) 1859-1872.

[2] A. Holmberg, M. Åbom, H. Bodén: Accurate experimental two-port analysis of flow generated sound. Journal of Sound and Vibration 33 6336-6354, 2011.

[3] C. Lahiri, K. Knobloch, F. Bake, L. Enghardt: Attenuation of sound in wide ducts with flow at elevated pressure and temperature. Journal of Sound and Vibration, 333 (2014) 3440-3458.

[4] G. Kirchhoff: Ueber den Einfluss der Wärmeleitung in einem Gase auf die Schallbewegung. Annalen der Physik 210 (1868) 177-193.

[5] R. E. Beatty: Boundary layer attenuation of higher order modes in rectangular and circular tubes. The Journal of the Acoustical Society of America 22 (1950) 850-854.

[6] M. Bruneau, Ph. Herzog, J. Kergomard, J. D. Polack: General formulation of the dispersion equation in bounded visco-thermal fluid, and application to some simple geometries. Wave Motion 11 (1989) 441-451.

[7] Y. Aurégan, R. Starobinski, V. Pagneux: Influence of grazing flow and dissipation effects on the acoustic boundary conditions at a lined wall. The Journal of the Acoustical Society of America 109 (2001) 59-64.

[8] S. W. Rienstra, M. Darau: Boundary-layer thickness effects of the hydrodynamic instability along an impedance wall. Journal of Fluid Mechanics 671 (2011) 559-573.

[9] E. J. Brambley: Acoustic implications of a thin viscous boundary layer over a compliant surface or permeable liner. Journal of Fluid Mechanics 678 (2011) 348-378.
[10] E. Dokumaci: On the effect of viscosity and thermal conductivity on sound propagation in ducts: A re-visit to the classical theory with extensions for higher order modes and presence of mean flow. Journal of Sound and Vibration 333 (2014) 5583-5599.

[11] D. Khamis, E. J. Brambley: Analytic model and concise impedance boundary condition for viscous acoustics in ducted shear flow. 22nd AIAA/CEAS Aeroacoustics Conference, No. AIAA 2016-2976, Lyon, France, May 2016.

[12] A. H. Nayfeh: Effect of the acoustic boundary layer on the wave propagation in ducts. The Journal of the Acoustical Society of America 54 (1973) 1737-1742.

[13] M. K. Myers: On the acoustic boundary condition in the presence of flow. Journal of Sound and Vibration 71 (1980) 429-434.

[14] H. Schlichting, K. Gersten: Boundary-Layer Theory. Springer, Berlin, 8th edition, 2000.

[15] E. Dokumaci: Sound transmission in narrow pipes with superimposed uniform mean flow and acoustic modelling of automobile catalytic converters. Journal of Sound and Vibration 182 (1995) 799-808.

[16] P. O. A. L. Davies: Practical flow duct acoustics. Journal of Sound and Vibration 124 (1988) 91-115.

[17] S. Sack, M. Åbom, C. F. Schram, K. Kucukcoskun: Generation and scattering of acoustic modes in ducts with flow. 20th AIAA/CEAS Aeroacoustics Conference, No. AIAA 2014-3115, Atlanta, GA, Jun 2014.

[18] T. Yazaki, Y. Tashiro, T. Biwa: Measurements of sound propagation in narrow tubes. Proceedings of the Royal Society of London A: Mathematical, Physical and Engineering Sciences 463 (2007) 2855-2862.

[19] C. Weng, S. Boij, A. Hanifi: On the calculation of the complex wavenumber of plane waves in rigid-walled low-Mach-number turbulent pipe flows. Journal of Sound and Vibration 354 (2015) 132-153.

[20] D. Khamis, E. J. Brambley: The effective impedance of a finite-thickness viscothermal boundary layer over an acoustic lining. 21st AIAA/CEAS Aeroacoustics Conference, No. AIAA 2015-2229, Dallas, TX, Jun 2015. 